\title{
Comparative Analysis of Classical and Quantum Plasmons in Graphene Nanostructures
}

\author{
Jian Wei You \\ Deppartment of Electronic and \\ Electrical Engineering, \\ University College London \\ London, United Kingdom \\ j.you@ucl.ac.uk
}

\author{
Nicolae C. Panoiu \\ Deppartment of Electronic and \\ Electrical Engineering, \\ University College London \\ London, United Kingdom \\ n.panoiu@ucl.ac.uk
}

\begin{abstract}
We investigate the physical properties of classical and quantum plasmons in graphene nanostructures using finitedifference time-domain computations and time-dependent density functional theory, respectively. The results show that the optical properties of quantum plasmons are markedly different from those of classical ones, both qualitatively and quantitatively.
\end{abstract}

Keywords-Plasmons, Graphene, Quantum Edge Effects, FDTD, TDDFT.

\section{INTRODUCTION}

In the past decade, graphene has been playing an increasingly important role in classical and quantum plasmonics [1-3], mainly due to its strong interaction with light, its potential for unprecedented optical field confinement, and advancements in nanofabrication techniques that allow one to create graphene structures with nanometer-size features. Inheriting these remarkable properties from the extended graphene sheet, graphene nanostructures provide an ideal platform for studying the physical properties of localized surface plasmons in nanometer-size plasmonic systems. In addition, quantum effects become increasingly important as the size of graphene dots is scaled down. This has been observed in relation to quantum plasmons of metallic nanoparticles [4,5]. However, graphene nanostructures provide a much more suitable testing ground for an in-depth investigation of key aspects of the classical-toquantum transition of localized plasmons. In this context, we systematically study the classical and quantum plasmonics in graphene nanostructures via the finite-difference time-domain (FDTD) method [6] and time-dependent density functional theory (TDDFT) [7]. This work lead to novel design rules for metamaterials based on molecular graphene nanodevices.

\section{COMPUTATIONAL APPROACH}

\section{A. Classical Plasmons - FDTD}

The classical plasmons in graphene nanostructures can be studied using the FDTD method, which is a general and accurate numerical method to solve Maxwell's equations. In FDTD simulations, the linear properties of graphene are generally described by the Kubo's formula for the surface conductance. Within the random-phase approximation [8], this formula can be

ERC Grant Agreement no. ERC-2014-CoG-648328 reduced to the sum of inter-band and intra-band contributions as

$$
\sigma_{s}\left(\omega, \mu_{c}, \tau, \mathrm{T}\right)=\sigma_{\text {intra }}\left(\omega, \mu_{c}, \tau, \mathrm{T}\right)+\sigma_{\text {inter }}\left(\omega, \mu_{c}, \tau, \mathrm{T}\right)
$$

where, the symbol $\omega$ is the angular frequency, $\mu_{c}$ is the chemical potential, $\tau$ is the relaxation time, $T$ is the Kelvin temperature. Based on (1), graphene can be modelled in FDTD simulations as a homogeneous medium with a specific conductivity.

\section{B. Quantum Plasmons - TDDFT}

The quantum plasmons of graphene nanostructures can be investigated using the TDDFT formalism, which is one of most reliable approaches to solve the Schrodinger equation. In this paper, we characterize the quantum dynamics in graphene nanostructures by computing its dynamical polarizabilities as

$$
\alpha_{x x}(\omega)=\mu_{x}(\omega) / E_{x}(\omega)
$$

where, $\mu_{x}(\omega)$ and $E_{x}(\omega)$ are the Fourier transformed electric dipole moment and electric field, respectively, which are both calculated using the TDDFT method.

\section{RESULTS AND DISCUSSION}

Using the FDTD and TDDFT methods, the optical spectra of classical and quantum plasmons of graphene with hexagonal, triangular, and stripe-like shapes were studied. First, we investigated the plasmon resonance energies for hexagonal and triangular graphene nanoflakes [9] as shown in Fig.1, where the frequency dispersion of quantum plasmon resonances determined using TDDFT and the classical ones calculated using the FDTD method are presented. This figure, shows that the plasmon resonance energies decrease as the size of the nanodisks increases, in both classical and quantum cases. Importantly, however, there two key differences between the classical and quantum regimes. First, the slope of the dispersion curves in the classical case is independent of the shape of the graphene nanoflake, whereas, in the quantum regime the corresponding slope vary with the GNF shape. Secondly, in the quantum regime, the dispersion curves of low- and high-energy plasmon resonances have different slopes, in contrast to the firstand second-order classical plasmons depicted with blue and green markers in Fig. 1, respectively. 


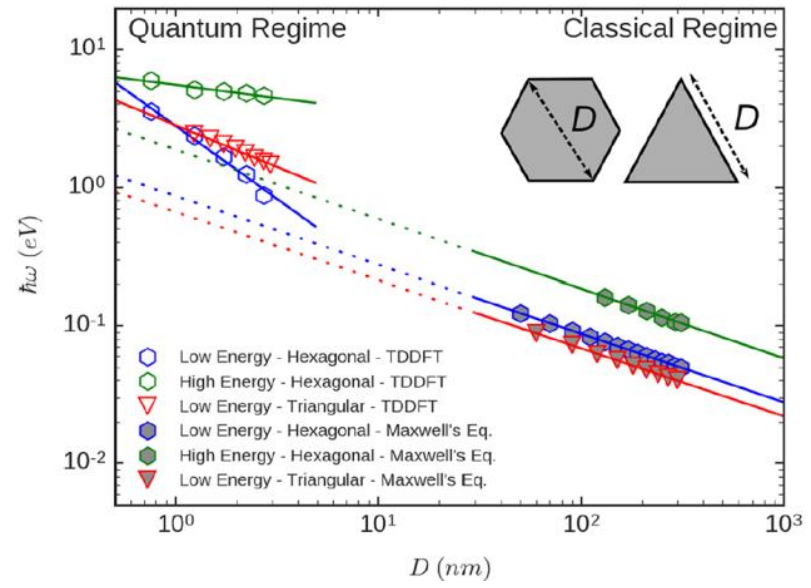

Fig. 1. Comparison of the size-dependence of the classical and the quantum plasmon resonance energies for hexagonal and triangular graphene nanodisks.

These differences suggest that quantum effects play an important role when the size of structure is very small. In fact, these quantum effects are mainly induced by the edge charge distribution. To illustrate this, the charge distribution of a graphene nanoribbon is computed using the TDDFT method, the corresponding results being summarized in Fig.2.
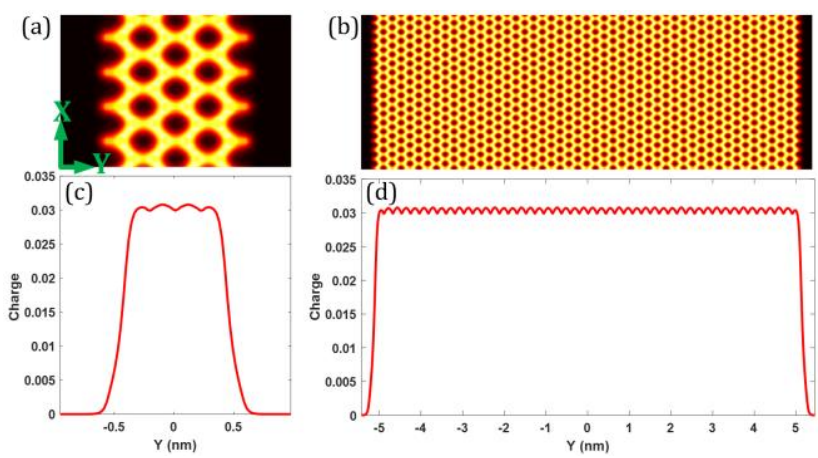

Fig. 2. a), b) Charge distribution of graphene nanorribons with different width. c), d) Dependence of charge distribution on the transverse coordinate, determined for graphene nanorribons with different width.

In Figs. 2(a) and 2(b), the width of ribbon is $W=1 \mathrm{~nm}$ and $W=10 \mathrm{~nm}$, respectively. The corresponding charge distribution along the $y$-direction is given in Figs. 2(c) and 2(d). These results show that the charge distribution at the ribbon edge is very different from that in the bulk region of the ribbon. It implies that the effective conductivity at the edge cannot be described by that in the bulk region. More importantly, recent studies [10] show that quantum edge effects can completely change the optical response of metallic nanoparticles. In order to verify this in the case of graphene, we varied the width of a nanoribbon, and calculated the corresponding classical and quantum plasmonic resonance energies. The edge effects can be fully incorporated in quantum simulation, whereas they are neglected in usual classical simulations.

The numerical results are given in Fig. 3, and show that there is a significant difference between the classical and quantum results for small ribbons. Furthermore, this difference decreases as the width of the ribbon increases, that is consistent with the variation of the ratio of the edge and bulk parts. Moreover, the quantum results eventually converge to the classical ones, which further proves that the difference between classical and quantum results is mainly due to edge effects as the ratio of the edge and bulk parts converges to zero in the limit of infinitely large width.

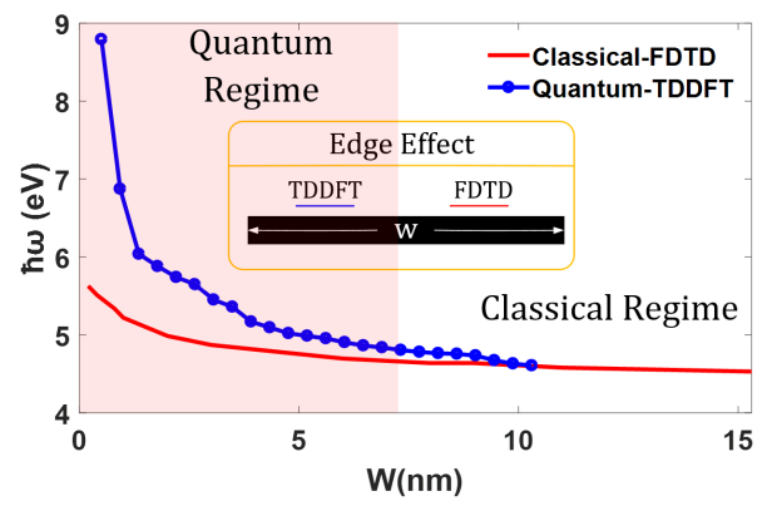

Fig. 3. Comparison of the size-dependence of classical and the quantum plasmon resonance energies for graphene nanoribbons with varying width, $W$.

\section{COCLUSIONS}

In conclusion, we have demonstrated that there are several key differences between the properties of classical and quantum plasmons in nano-sized graphene structures. After analysing the optical properties of classical and quantum plasmons, calculated using FDTD and TDDFT methods, respectively, we conclude that edge effects in such nanostructures are the main reason for these difference. This finding can guide the development of a quantum correction model to bridge the description of classical and quantum plasmons employed in practical nanodevices.

\section{REFERENCES}

[1] F. H. L. Koppens, D. E. Chang, F. J. G. de Abajo, "Graphene plasmonics: a platform for strong light-matter interactions," Nano Lett. vol. 11, pp. 3370-3377, 2011.

[2] J. D. Cox and F. J. G. de Abajo, "Electrically tunable nonlinear plasmonics in graphene nanoislands," Nat. Commun., vol. 5, pp. 5725, 2014.

[3] J. W. You and N. C. Panoiu, "Polarization control using passive and active cross graphene gratings," Opt. Express, vol. 26, pp. 1882-1894, 2018.

[4] J. A. Scholl, A. L. Koh, J. A. Dionne, "Quantum plasmon resonances of individual metallic nanoparticles," Nature, vol. 483, pp. 421-427,2012.

[5] K. J. Savage, M. M. Hawkeye, R. Esteban, A. G. Borisov, J. Aizpurua, J. J. Baumberg, "Revealing the quantum regime in tunnelling plasmonics," Nature, vol. 491, pp. 574-577, 2012.

[6] A. Taflove and S. C. Hagness, Computational Electrodynamics: The Finite-Difference Time-Domain Method, 3rd ed. Artech House, 2005.

[7] M. A. L. Marques, N. Maitra, F. Nogueira, E. K. U. Gross, A. Rubio, Fundamentals of Time-Dependent Density Functional Theory, Springer Berlin Heidelberg, New York, 2012.

[8] G.W. Hanson, "Dyadic Green's functions and guided surface waves for a surface conductivity model of graphene," J. Appl. Phys., vol. 103, pp. 064302, 2008.

[9] D. Z. Manrique, J. W. You, H. Deng, F. Ye, N. C. Panoiu, "Quantum plasmon engineering with interacting graphene nanoflakes," J. Phys. Chem. C, vol. 121, pp. 27597-27602, 2017.

[10] W. Yan, M. Wubs, N. A. Mortensen, "Projected dipole model for quantum plasmonics, ” Phys. Rev. Lett., vol. 115, p.137403, 2017. 